\title{
YiQi Tongluo Granule against Cerebral Ischemia/Reperfusion Injury in Rats by Freezing GluN2B and CaMK II through NMDAR/ERK1/2 Signaling
}

\author{
Si-peng Wu, ${ }^{a, b, c, \#}$ Dan Li, ${ }^{d, \#}$ Ning Wang, ${ }^{* a, b, c}$ Jin-cai Hou, ${ }^{*, c}$ and Li Zhao ${ }^{a, b, c}$ \\ ${ }^{a}$ Anhui Province Key Laboratory of Chinese Medicinal Formula, Anhui University of Chinese Medicine; Hefei \\ 230012, China: ${ }^{b}$ Institute for Pharmacodynamics and Safety Evaluation of Chinese Medicine, Anhui Academy of \\ Chinese Medicine; Hefei 230012, China: ${ }^{c}$ Key Laboratory of Xin'an Medicine, Ministry of Education; Hefei 230012, \\ China: and ${ }^{d}$ Jing-Jin-Ji Joint Innovation Pharmaceutical (Beijing) Co., Ltd.; Beijing 100083, China. \\ Received October 15, 2018; accepted December 19, 2018; advance publication released online December 28, 2018
}

Yiqi Tongluo Granule (YQTL) is a kind of proprietary Chinese medicine, manufactured by China Shineway Pharmaceutical Group Ltd., under the authority of China Food and Drug Administration (CFDA) treating cardiovascular and cerebrovascular diseases such as ischemic stroke in China, however the underlying mechanism of YQTL on treating ischemic stroke has not been revealed. This study is aimed to evaluate the protective effect of YQTL on cerebral ischemia/reperfusion (I/R) injury and inquire into its underlying mechanisms. Cerebral $\mathrm{I} / \mathrm{R}$ injury was induced by occluding the middle cerebral artery for $2 \mathrm{~h}$ followed by $24 \mathrm{~h}$ reperfusion. And regional cerebral flow was monitored by Laser Doppler flow during ischemia phase. The infarct volume was evaluated by Triphenyte-trazolium chloride staining. The protective effects of YQTL were assessed by a number of parameters, including neurological scores, regional cerebral blood flow, pathological changes of neuron in hippocampuses and hippocampus calcium level. The proteins of extracellular signal-regulated kinase (ERK), $N$-methyl D-aspartate receptor subtype 2B (GluN2B) and p-calcium-dependent protein kinaseII (CaMKII) response were assayed by Western blotting. I/R caused significant change in neurological deficit scores, regional cerebral flow and infarct volume. However results in YQTL groups and Nimodipine Tablets (NMDP) group were reversed. Subsequently YQTL reduced I/R-induced calcium influx. Results of hematoxylin-eosin staining manifested that YQTL significantly improved neuronal injury after $I / R$ in rats. Meanwhile, microdialysis data demonstrated that extracellular glutamate was increased in the striatum during ischemia reperfusion, which was reduced by YQTL. YQTL and mitogen-activated protein extracellular kinase (MEK) inhibitor suppressed the I/R-mediated over-expression of GluN2B, p-ERK, ERK and p-CaMKII proteins expression. Putting these together, our results suggest that YQTL played a neuroprotective role in cerebral $\mathrm{I} / \mathrm{R}$ injury, which might be exerted by inhibiting the excitotoxicity and expression of GluN2B, p-CaMKII and MEK/ERK signal pathway.

Key words Yiqi Tongluo Granule; cerebral ischemia reperfusion injury; excitotoxicity; mitogen-activated protein extracellular kinase/extracellular signal-regulated kinase signaling

\section{Introduction}

Ischemia stroke accounts for approximately $80 \%$ of all stroke cases, and has been taken into account as a major cause of disability and death. ${ }^{1)}$ Despite the fact that cerebral reperfusion is seen as an essential mechanisms for the recovery of brain injury caused by ischemia, recent studies suggested otherwise. Indeed, numerous studies have highlighted the detrimental effects of reperfusion to the brain due to enhanced neuronal inflammation, oxidative stress and calcium overload. ${ }^{2,3)}$

Considerable evidence has indicated that excessive excitatory amino acids (EAA) released after ischemia reperfusion plays a crucial role in ischemia/reperfusion (I/R) injury. ${ }^{4,5}$ Ionic glutamate $N$-methyl-D-aspartic acid receptors (NMDAR) is one of the major receptors responsible for the acute excitotoxicity during $\mathrm{I} / \mathrm{R}$, and they are ligand-gated ion channels with a high permeability to calcium. ${ }^{6)}$ Excitotoxicity and calcium overload are two major pathologic hallmarks of ischemia stroke, which are mediated by the NMDARs. ${ }^{7)}$ During isch-

\footnotetext{
${ }^{\#}$ These authors contributed equally to this work.

emia, the $N$-methyl D-aspartate receptor subtype 2B (GluN2B) is phosphorylated by the excessive release of glutamate (Glu) in the ischemia site, causing an influx of $\mathrm{Ca}^{2+}$ into the neurons. However, the increase of intracellular $\mathrm{Ca}^{2+}$ level leads to the activation of the extracellular signal-regulated kinase 1 and 2 protein (ERK1/2) signaling pathway by binding to a number of signaling molecules such as the protein tyrosine kinase (PTK), protein kinase A (PKA) and calcium-dependent protein kinaseII (CaMKII) binding protein. Activation of the ERK1/2 signaling pathway has been shown a vital role in neuronal death during cerebral ischemia, hence, suppress this pathway could be beneficial for the management of ischemia stroke. $^{8)}$

Traditional Chinese Medicine (TCM) has been extensively employed in the prevention and treatment of cerebral ischemia. ${ }^{9)}$ Yiqi Tongluo Granule (YQTL) is a kind of proprietary Chinese medicine, manufactured under the authority of China Food and Drug Administration (CFDA). It can treat various cardiovascular diseases, such as cerebral ischemia, coronary heart disease and hypertension. ${ }^{10)}$ Although the beneficial cardiovascular effect has been demonstrated in a number of 
clinical trials, its neuroprotective effect and the underlying mechanisms against cerebral I/R have not been fully explored. Therefore, this study is designed to evaluate the neuroprotective effects of YQTL using middle cerebral artery occlusion (MCAO) method. In addition, the role of the NMDAR/ ERK1/2 signaling was also investigated.

\section{Experimental}

Reagents YQTL was provided by Shineway Pharmaceutical Co., Ltd. (National number: Z20120001, Hebei, China). Nimodipine Tablets (NMDP) was issued by Bayer (Batch number: H20003010). Fluo-3/AM, triphenyltetrazolium chloride (TTC), hematoxylin-eosin staining (HE) were acquired from Sigma Chemical Co. (U.S.A.). Glu and $\gamma$-aminobutyric acid (GABA) standard (batch number: BCBQ3296V and BCBN3020V) was purchased from Sigma. Methanol and acetonitrile were chromatographic grade, and the remaining reagents were analytical grade. U0126 was purchased from Selleck Chemicals Co., Ltd. (London, Canada). Rat antibodies against GluN2B, p-CaMKII, ERK, p-ERK, $\beta$-actin were recruited from Abcam (Cambridge, MA, U.S.A.). Horseradish peroxidase (HRP) conjugated goat anti-rat immunoglobulin $\mathrm{G}$ (IgG) were purchased from Santa Cruz Biotechnology (Santa Cruz, CA, U.S.A.). All of the other chemicals and reagents were standard, commercially available.

Animals Adult male Sprague Dawley (SD) rats (body weight, 240-260g) were provided by Jested Laboratory Animal Co., Ltd. Certificate number: (SCXK: Shanghai, 2013-0006). All experiments were approved by the Animal Care and Use Committee of Anhui University of Chinese Medicine and performed in accordance with the Guidelines of the National Institutes of Health on the Care and Use of Animals.

Cerebral I/R Model Cerebral I/R injury model was established according to Longa's ${ }^{11)}$ methods. Rats were anesthetized with isoflurane (3-4\% for anesthesia induction; $1-2 \%$ for anesthesia maintenance) before, exposing the left common carotid artery (CCA). The left CCA and the internal carotid artery (ICA) were blocked with microvascular aneurysm clips, followed by occluding the middle cerebral artery (MCA) by inserting a nylon filament (Beijing Sunbio Biotech, Beijing, China) coated through the external carotid artery (ECA) $(18 \pm 2 \mathrm{~mm})$. Two hours later, the filament was removed to achieve reperfusion. Sham-operate rats received the same procedures as the Model group, but no filament was inserted to the MCA. Temperature of the operation chamber was maintained at $37 \pm 0.5^{\circ} \mathrm{C}$ throughout the whole surgery. The regional cerebral blood flow ( $\mathrm{rCBF}$ ) was monitored by laser Doppler flow (LDF, PeriFlux5000 Perimed Co., China).

Experimental Groups and Drug Administration The experimental protocol for the current study was showed in Fig. 1. Firstly, to explore the protective effect of YQTL on rats with cerebral I/R injury, rats were randomly divided into four groups: Sham group, Model group, YQTL groups (1.8, $3.6 \mathrm{~g} / \mathrm{kg}$ ) group and Positive group (NMDP, $0.02 \mathrm{~g} / \mathrm{kg}$ ). Dosages of YQTL $(3.6 \mathrm{~g} / \mathrm{kg})$ and NMDP $(0.02 \mathrm{~g} / \mathrm{kg})$ were equivalent dose of clinical adult patients. Twenty-four hours after ischemia reperfusion, rats in YQTL group and NMDP group were given drug by intragastric administration of their respective dose, once daily for $14 \mathrm{~d}$, and the Sham and Model groups were given physiological saline. Secondly, to ascertain the effect of YQTL on amino acids in rats induced by I/R (experiment 2). Rats were randomly divided into four groups: Sham group, Model group, YQTL groups $(1.8,3.6 \mathrm{~g} / \mathrm{kg})$ group and Positive group (NMDP, $0.02 \mathrm{~g} / \mathrm{kg}$ ). Rats in YQTL group and NMDP group were given drug by intragastric administration of their respective dose, once daily for $7 \mathrm{~d}$ before MCAO, and the Sham and Model group was given physiological saline. The content of Glu and GABA at ischemia $2 \mathrm{~h}$ or reperfusion $24 \mathrm{~h}$ time point detected by microdialysis.

In order to investigate whether MEK/ERK signal pathway and NMDA receptor were participated in YQTL
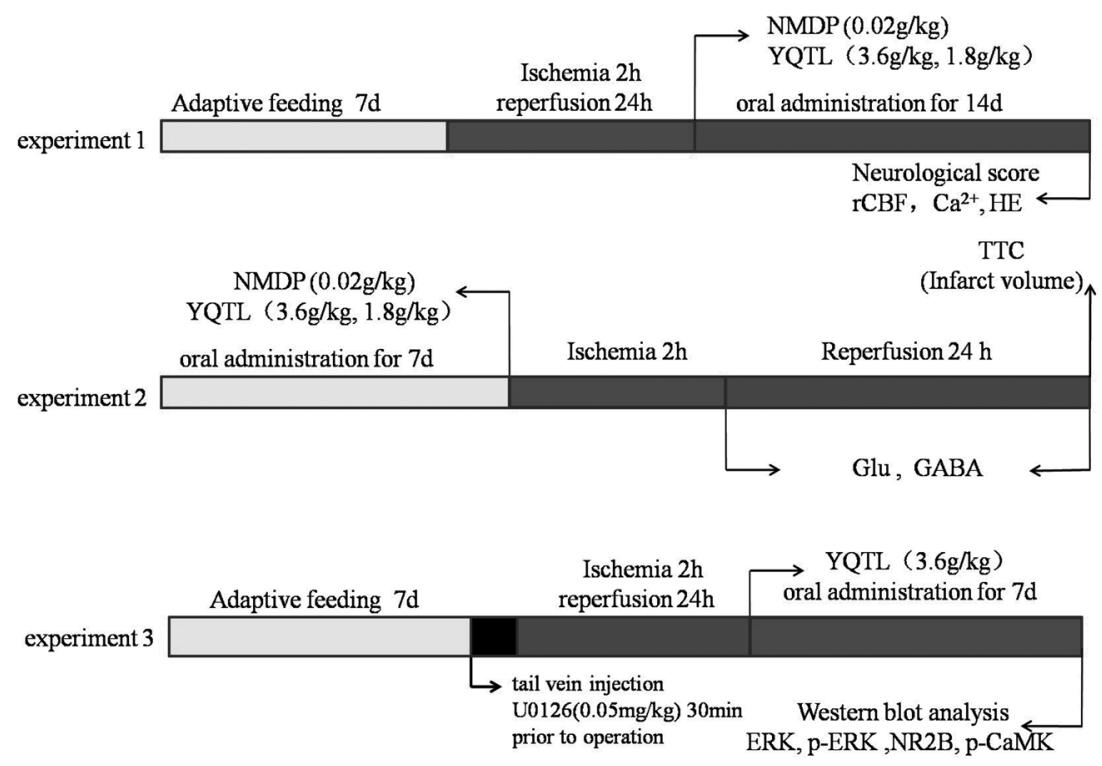

Fig. 1. Schematic Diagrams of the Experimental Protocol

As shown in this experiment 1 , the rats were adaptive feeding for $7 \mathrm{~d}$ before ischemia $2 \mathrm{~h}$ follow reperfusion $24 \mathrm{~h}$, rats were treated with YQTL or NMDP for $7 \mathrm{~d}$. Then rats were treated with YQTL or NMDP for $7 \mathrm{~d}$ prior to I/R in experiment 2. Finally, in experiment 3, U0126 (0.5 mg $/ \mathrm{kg}) \mathrm{was}$ tail vein injection $30 \mathrm{~min}$ prior to I/R. After $24 \mathrm{~h}$ reperfusion, rats were treated with YQTL or NMDP for $7 \mathrm{~d}$. At the indicated time point rats were harvested for subsequent experiments. 
protect against cerebral $\mathrm{I} / \mathrm{R}$, rats were randomly divided into five groups: Sham group, Model group, YQTL group $(3.6 \mathrm{~g} / \mathrm{kg}), \quad \mathrm{U} 0126(0.5 \mathrm{mg} / \mathrm{kg})$ group and Combined group (YQTL + U0126). After reperfusion, YQTL group and YQTL + U0126 combine group was given drug by intragastric administration of their respective dose, once daily for $7 \mathrm{~d}$, Sham, Model and U0126 groups were given physiological saline for $7 \mathrm{~d}$. U0126 (final dose: $0.5 \mathrm{mg} / \mathrm{kg}$ ) used for administration of caudal vein injection to rats 30 min prior to operation ${ }^{12)}$ (experiment 3). All experiments were conducted in triplicate.

Neurological Score Neurological scoring was assessed at 1st day, 7th day and 14th day after MCAO according to the scales described by Longa et al. ${ }^{11)}$ No neurologic deficit $=$ score 0 , failure to extend forepaw fully $=$ score 1 , circling to the left $=$ score 2 , falling to the left or no spontaneous motor activity $=$ score 3 , do not walk spontaneously and experience a lowered stage of consciousness $=$ score 4 .

Hematoxylin-Eosin (HE) Staining Rats were anesthetized with anesthetized with isoflurane (3-4\% for anesthesia induction; $1-2 \%$ for anesthesia maintenance) and $4 \%$ paraformaldehyde (Sigma Chemical Co.) was injected into, the left ventricle via a needle. Brains were removed and post-fixed in $4 \%$ paraformaldehyde at $4^{\circ} \mathrm{C}$. They were dehydrated in graded ethanol and cleared in xylene, before being embedded in paraffin. Coronal sections ( $5 \mu \mathrm{m}$ thick) were gathered and stained with HE.

Monitoring Local Blood Flow Changes by Laser Doppler Flow rCBF of the corpus striatum ipsilateral to the cerebral infarction were measured using LDF. In brief, rats were anesthetized, disinfected and cut the skin and subcutaneous tissue along the right side of the skull. The $\mathrm{rCBF}$ was recorded in the following coordination relative to Bregma: $\mathrm{ML}:+2.0 \mathrm{~mm}$, AP: $+1.0 \mathrm{~mm}$ with a bio-rubber probe connected to the conventional debugging zero according to previous reports. ${ }^{13)}$ The cerebral blood flow decreased by $70 \%$ of the baseline during the cerebral ischemia phase, and returned to $80 \%$ of the baseline when reperfusion phase. Cerebral blood flow diagram confirmed successful establishment of MCAO model in rats.

Flow Cytometry Detect the Content of Calcium Half of the hippocampus was placed in a $4 \mathrm{~mL}$ centrifuge tube and disintegrated with eye surgery shearing mechanical force. The tissue was digested in $0.25 \%$ trypsin solution (Beyotime, China), in water bath at $37^{\circ} \mathrm{C}$ for $10 \mathrm{~min}$, centrifuged at $8^{\circ} \mathrm{C}$ for $5 \mathrm{~min}$ at $1500 \mathrm{rpm}$. Supernatant was discarded and $1 \mathrm{~mL}$ $20 \%(\mathrm{w} / \mathrm{v})$ fetal bovine serum (GIBCO, U.S.A.) was added to stop the reaction. The tissue was filtered using a 200 nylon meshes, and centrifuged at $1000 \mathrm{rpm}$ for $5 \mathrm{~min}$. The supernatant was discarded and $3 \mathrm{~mL}$ D-Hank's liquid (Leagene, China) was added to wash the precipitates, followed by centrifuging at $1000 \mathrm{rpm}$ for $5 \mathrm{~min}$. The supernatant was discarded and these washing steps were repeated twice; Fluo-3/AM working solution was added to stain $\mathrm{Ca}^{2+}$ for $30 \mathrm{~min}$ at $37^{\circ} \mathrm{C}$ in the dark. One milliliter of D-Hank's solution was added to cover the cells and brought them back to a single cell suspension. Finally, $\mathrm{Ca}^{2+}$ images were captured using a fluorescence microscope (Flow Cytomics TM FC500, U.S.A.).

TTC Staining Twenty four hours after reperfusion, cerebral infarct volume was evaluated using 2,3,5-triphenyltetrazolium chloride (TTC) staining (Sigma Chemical Co.). After $24 \mathrm{~h}$ reperfusion, brains were removed quickly and kept at $-20^{\circ} \mathrm{C}$ for further assessment. Frozen brains were sliced into uniform coronal sections of $1.5 \mathrm{~mm}$ thickness. The slices were incubated in $2 \%$ TTC ( $\mathrm{pH}=7.4$, Sigma Chemical Co.) solution at $37^{\circ} \mathrm{C}$ for $20 \mathrm{~min}$. Brain infarct volume was calculated by Image $\mathrm{J}$, multiplying this by the slice thickness $(1.5 \mathrm{~mm})$. The volume of infarction in each brain was calculated by Infarct size $\times$ average slice thickness $(2 \mathrm{~mm})$ and analyzed by Image $\mathrm{J}$.

Microdialysis We used microdialysis techniques to collect brain amino acids and analyzed the content of amino acids by HPLC-Fluorescence Detector (FLD) during I/R. SD rats were anesthetized by $3-4 \%$ isoflurane and perfused with $4 \%$ paraformaldehyde. Concentric microdialysis of probes $4 \mathrm{~mm}$ in length were lowered into the striatum using the following coordinates relative to Bregma: ML: $3.0 \mathrm{~mm}$, AP: $0.2 \mathrm{~mm}$ and $3.5 \mathrm{~mm}$ below. Microdialysis probes were continuously infused with the KRP buffer $(120 \mathrm{mM} \mathrm{NaCl}, 2.4 \mathrm{mM}$

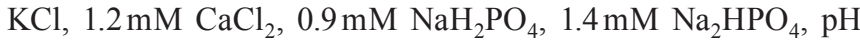
7.4) using a CMA infusion pump (CMA, Sweden). Each group samples were collected at time point to determine the content of Glu or GABA by HPLC-FLD.

Determination of Amino Acids by HPLC-FLD Determination of amino acids in microdialysis samples by high performance liquid chromatography equipped with a fluorescence detector. Analytes were separated by a Aglient Eclipse AAA $(4.6 \times 150 \mathrm{~mm}, 5 \mu \mathrm{m}$.) Samples were injected into the column and analysis was carried out at $35^{\circ} \mathrm{C}$. The mobile phase consisted of eluents A (buffer:methanol:tetrahydrofuran, 400: 95:5, v/v) and B (buffer: methanol, 120: 380, v/v) was used for separation, wherein the buffer is $20 \mathrm{mM}$ sodium acetate solution ( $\mathrm{pH}$ 7.2). The elution program was optimized and conducted as follows: $0-10 \mathrm{~min}, 0-63 \% \mathrm{~B} ; 10-12 \mathrm{~min}, 63 \% \mathrm{~B}$; $12-12.01 \mathrm{~min}, 63-100 \% \mathrm{~B} ; 12.01-17 \mathrm{~min}, 100 \% \mathrm{~B} ; 17-18 \mathrm{~min}$, $100-0 \% \mathrm{~B} ; 18-21 \mathrm{~min}, 0 \% \mathrm{~B}$. After a $10 \mathrm{~min}$ equilibration period, the samples were used for injection. The peaks were recorded using Shimadzu RF-20A fluorescence detector with excitation/emission peaks at $340 / 455 \mathrm{~nm}$ and the solvent flow rate was kept at $0.8 \mathrm{~mL} \cdot \mathrm{min}^{-1}$. The data were collected by INT7 card and the result was expressed in $\mu \mathrm{mol} / \mathrm{L}$.

Western Blotting Analysis Rats were randomly divided into five groups: Sham group, Model group, YQTL group $(3.6 \mathrm{~g} / \mathrm{kg}), \mathrm{U} 0126(0.5 \mathrm{mg} / \mathrm{kg})$ group and the combination group (YQTL $\left.{ }_{3.6 \mathrm{~g} / \mathrm{kg}}+\mathrm{U} 0126\right)$. GluN2B, ERK, p-ERK, $\beta$-actin and $\mathrm{p}$-CaMKII proteins expression level were assessed as described previously. The hippocampus tissue was homogenized with pre-cold RIPA lysis buffer and Immediately add $10 \mu \mathrm{L}$ each of phenylmethanesulfonyl fluoride (PMSF, Beyotime Institute of Biotechnology, Nanjing, China) and phosphatase inhibitor (1 mM) (Calbiochem, Merck, Germany), centrifuged at $12000 \mathrm{rpm}$ at $4^{\circ} \mathrm{C}$ for $15 \mathrm{~min}$. The supernatant was collected and protein concentration was quantified using the bicinchoninic acid (BCA) (Beyotime Institute of Biotechnology) method. Proteins samples were electrophoresed on a $12 \%(\mathrm{w} / \mathrm{v})$ sulfate-polyacrylamide gel electrophoresis (SDS-PAGE) gel, and transferred to a polyvinylidene difluoride (PVDF) membrane. The membrane was blocked with $5 \%(\mathrm{w} / \mathrm{v})$ skim milk for $2 \mathrm{~h}$ at room temperature, and immune blotted overnight at $4^{\circ} \mathrm{C}$ with the primary antibodies to GLUN2B (1:1000), ERK $(1: 500)$, p-ERK $(1: 500)$, p-CaMKII $(1: 1000)$ and $\beta$-actin $(1: 1000)$, respectively. After washing with Phosphate Buffered Saline-Twen-20 (PBST) for 3 times, the membrane was incubated in the HRP $(1: 5000)$ conjugated secondary antibody solution (goat anti-rat) was incubated for $2 \mathrm{~h}$ at $37^{\circ} \mathrm{C}$. Chemilu- 


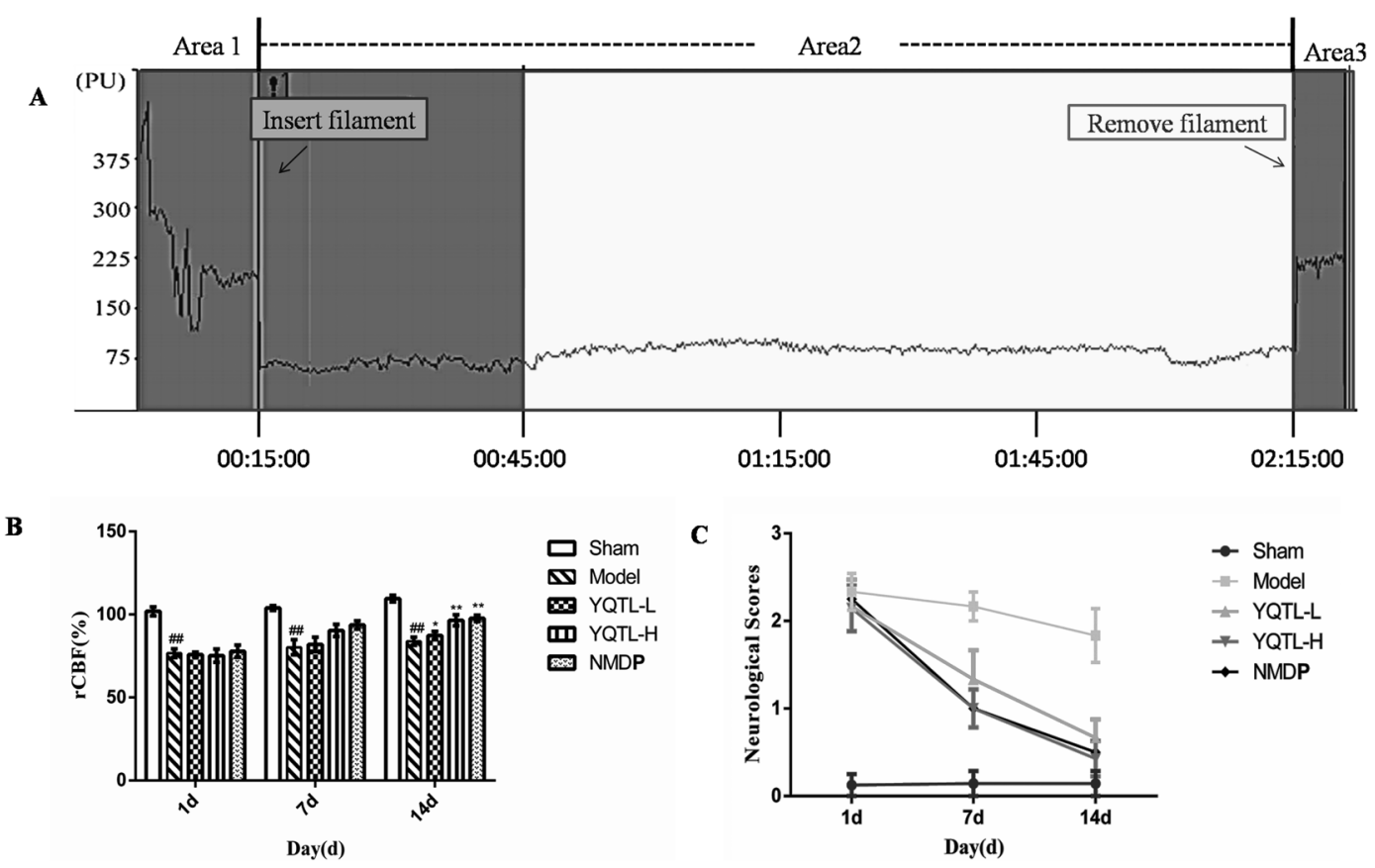

Fig. 2. Effects of YQTL on Blood Flow and Neurological Scores

(A) The dynamic change of rCBF during MCAO. Area 1 indicated the baseline of blood flow, this time was prepare time for operation. This text box of "insert filament" and "Remove filament" represents inserting and remove filament the blood vessel, respectively. The blood flow fell rapidly reaching about $30 \%$ of the baseline when the filament was inserted into the middle cerebral artery in area 2, the $2 \mathrm{~h}$ was ischemia time. (Area 3) $2 \mathrm{~h}$ later, the cerebral blood flow began recover to about $80 \%$ after removing the filament. (B) The changes of $\mathrm{rCBF}$ for each group at $1 \mathrm{~d}, 7 \mathrm{~d}$ and $14 \mathrm{~d}$. The $\mathrm{rCBF}$ of YQTL high dose group and NMDP group significantly recover on day 14. The value was relative to the baseline, not an absolute value. (C) Effect of YQTL on neurological scores. Sham: sham group; Model: Model group; YQTL-L: YQTL $(1.8 \mathrm{~g} / \mathrm{kg})$ group; YQTL-H: YQTL $(3.6 \mathrm{~g} / \mathrm{kg})$ group; NMDP: NMDP $(0.02 \mathrm{~g} / \mathrm{kg}) .{ }^{\#} p<0.01$ vs. Sham group; ${ }^{*} p<0.05,{ }^{* *} p<0.01$ vs. Model group. Data are presented as means \pm standard error of the mean (S.E.M.) $n=8$.

cent signal was developed using the enhanced chemiluminescence (ECL) reagents (Thermo, U.S.A.). Images were captured using an Amersham Imager 600 (General Electric, U.S.A.). $\beta$-Actin was used as an internal control.

Statistical Analysis All data were presented as mean \pm standard deviation (S.D.). Statistical analysis was performed using SPSS (SPSS, Inc., version 23.0.) Multi-group statistical difference was analyzed using ANOVA way. Differences between experimental groups were determined by Fisher's least significant difference (LSD) post-hoc test method, $p$-values $<0.05$ was considered as statistically significant difference.

\section{Results}

Cerebral Blood Flow and Neurological Score As shown in Fig. 2A, the blood flow fell rapidly after the filament was inserted into the middle cerebral artery, reaching $(24 \pm 2.94) \%$ of baseline in areal. Two hours later, the blood flow began to recover $(85.33 \pm 6.18) \%$ after removing the filament as indicated in area3. Then we monitored cerebral blood flow on days 1,7 and 14 after treatment. It was found that the rCBF of the Model group and the treatment groups was lower than that of the Sham group at 1st day. There was increase in $\mathrm{rCBF}$ in the model group and treatment group throughout the experimental period as observed at days 7 and 14. However, the rCBF of the model group was still significantly reduced to the sham group at day $14(p<0.01)$. In contrast, NMDP and YQTL group $(3.6 \mathrm{~g} / \mathrm{kg})$ significantly restored the $\mathrm{CBF}$ at day 14 when compared the Sham group (Fig. 2B).

Neurological scores were significantly increased in the Model group compared to the sham group throughout the $14 \mathrm{~d}$ experimental period. NMDP drastically reduced the neurological score as observed at days 7 and 14, in a time-dependent manner. Similarly, both low and high doses of YQTL significantly reduced the neurological score in a time-dependent manner (Fig. 2C).

Effects of YQTL on Pathological Changes Neuron histological changes in the hippocampus CA1 region were assessed by HE staining ( $\times 200$ magnification). The hippocampus CA1 from the sham group exhibited normal morphology (Fig. 3A). In contrast, significant interstitial edema and swelling of nerve cells were observed in the model group (Fig. 3B). Moreover, neuron cells were severely deformed, nucleus was pyknotic and tissues were necrotic. YQTL treatment remarkably attenuated the I/R-induced neuronal damage (Figs. 3C, D). The cells were tightly arranged with distinct outline. Fewer cells swelling and interstitial edema were observed in the YQTL high dose and NMDP group (Figs. 3D-E).

Drug Effect on Content of Calcium To determine the effect of YQTL on the content of $\mathrm{Ca}^{2+}$ induced by I/R, flow cytometry was performed to detect $\mathrm{Ca}^{2+}$. As showed in Figs. $4 \mathrm{~A}, \mathrm{~B}$, the content of $\mathrm{Ca}^{2+}$ rose to $91 \%$ with $\mathrm{I} / \mathrm{R}$, which was significantly higher than the Sham group (29.3\%). However, the content of $\mathrm{Ca}^{2+}$ was significantly lower in NMDP group (51.1\%) compared with the model group (Fig. 4E). The content of $\mathrm{Ca}^{2+}$ in YQTL groups was 85.6 and $41.2 \%$, respectively, in a concentration-dependent manner. These results suggested that YQTL inhibited calcium influx in the hippocampus after I/R injury (Figs. 4C, D).

Effect on Cerebral Infarct Volume No brain infarction was observed in the Sham group. A large infarct volume was observed in both the striatum and the adjacent cortex in 


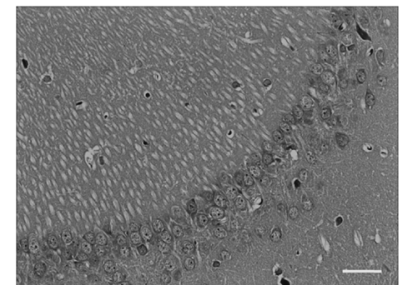

A

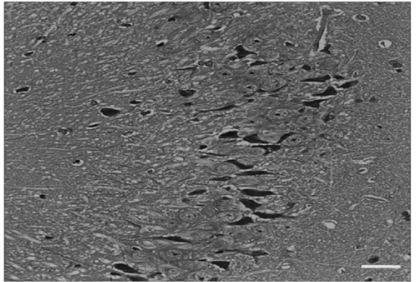

C

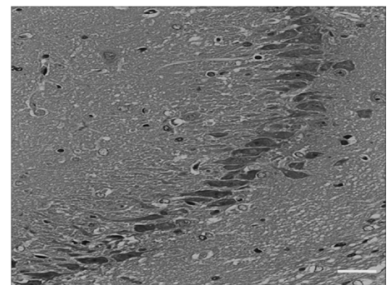

B

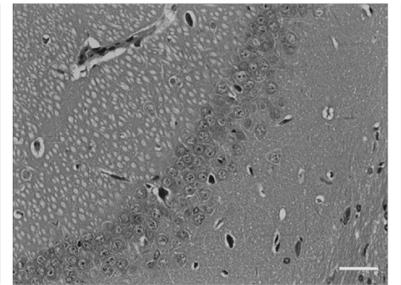

D

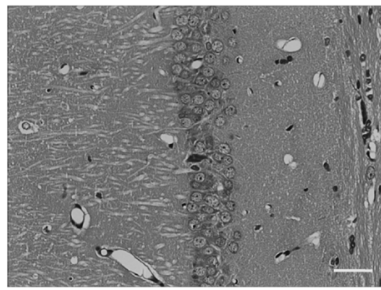

E

Fig. 3. HE Stains of Hippocampus CA1 Region Neurons

(A) Sham group; (B) Model group; (C) YQTL (1.8g/kg) group; (D) YQTL (3.6g/kg) group; (E) NMDP (0.02 g/kg) group. The Pathological Damage of hippocampus CA1 region neurons. HE staining had light red staining in the cytoplasm and spotty light blue staining in the nucleus indicated that the neurons were normal. The cells were seedless and arranged loosely, obvious cell edema was seen to represent cell damage. All the experiments were performed in triplicate. Scale bar $=100 \mu \mathrm{m}(\times 200$ magnification).

a

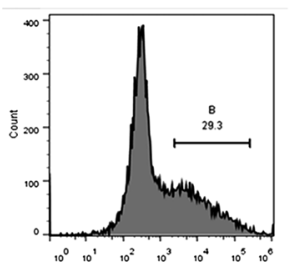

$\mathbf{A}$

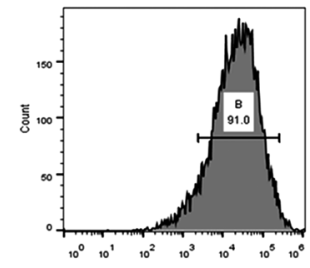

$\mathbf{B}$

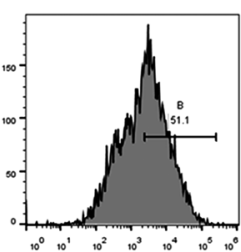

E

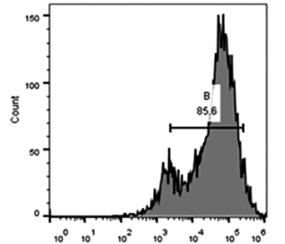

C

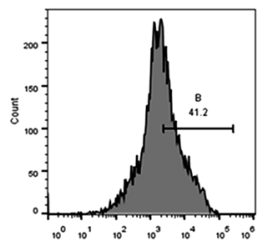

D $\mathrm{b}$

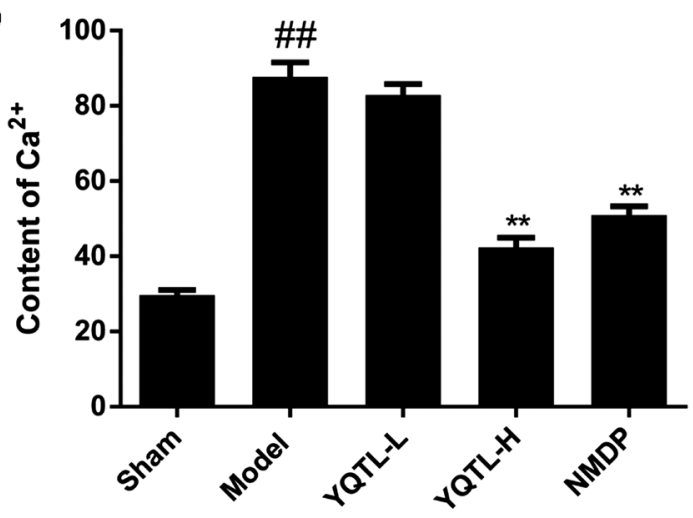

Fig. 4. Effects of YQTL on $\mathrm{Ca}^{2+}$ in the Hippocampus

(a) Calcium fluorescence intensity of each group. (A) Sham group, (B) Model group, (C) YQTL (1.8g/kg) group, (D) YQTL (3.6g/kg) group, (E) NMDP (0.02 g/kg) group. Fluorescence intensity represents the content of $\mathrm{Ca}^{2+}$, and Sham group was used as benchmark for each group. (b) Quantitative analysis of Ca ${ }^{2+}{ }^{\# \#} p<0.01 v s$. Sham group; $* * p<0.01 v s$. Model group. Data are presented as means \pm S.E.M. $n=8$.

the Model group. Infarct volume was significantly reduced in YQTL-H groups. NMDP also significantly decreased the infarct volume (Fig. 5A). As shown in Fig. 5B, infarct volume significantly increased to $(33.9 \pm 1.63) \%$ at the $24 \mathrm{~h}$ of reperfusion in Model group ( $p<0.01$ versus Sham group), YQTL high dose reduced the infarct volume to $(12.53 \pm 0.98)$ and NMDP group reduced to $(10.26 \pm 1.22) \%(p<0.01$ versus Model group).

Effect of YQTL on Amino Acids In the sample, the concentration of amino acid as horizontal coordinates and the corresponding peak area as vertical coordinate, the regression equation is used to calculate the standard curve. The standard curves of Glu (Fig. 6I) and GABA (Fig. 6II) were $Y=4.447 X+306.2 \quad\left(R^{2}=0.998\right), \quad Y=18.68 X+294.8$ $\left(R^{2}=0.996\right)$, respectively, the correlation coefficient is higher than 0.9 and have good linear relation. As show in Fig. 6, under the established HPLC conditions, the Glu retention time of the standard was about $11.4 \mathrm{~min}$ and the GABA retention time was about $29.1 \mathrm{~min}$. The separation was better with other chromatographic peaks and the response values were higher (Figs. 6A, B). In the retention time of $11 \mathrm{~min}$ and $29-30 \mathrm{~min}$, liquid chromatogram of microdialysis fluid contained YQTL appeared a peak with the same retention time as Glu and GABA standard (Fig. 6C).

Glu content increased rapidly with over $400 \%$ increase in ischemia $2 \mathrm{~h}(p<0.01 v s$. Sham group), but the increase of Glu is reversed by YQTL or NMDP. High dose YQTL $(3.6 \mathrm{~g} / \mathrm{kg})$ and NMDP $(0.2 \mathrm{~g} / \mathrm{kg})$ significantly reduced Glu to $50.3 \pm 1.56$ and $31.5 \pm 0.70 \mu \mathrm{mol} / \mathrm{L}$, respectively, at $24 \mathrm{~h}$ of reperfusion compared with Model group rats ( $p<0.01$ vs Model group) (Table 1). As show in Table 2, the GABA content rapidly increased to $(17 \pm 1) \mu \mathrm{mol} / \mathrm{L}$ during ischemia $2 \mathrm{~h}(p<0.01 v s$ Sham group), YQTL groups and NMDP group remarkably increased GABA at reperfusion $24 \mathrm{~h}$ than Model group, and 
A

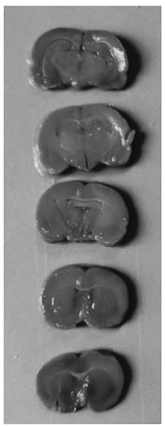

Sham

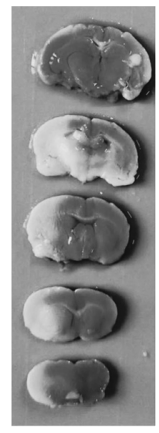

Model

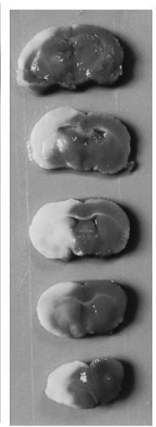

YQTL-L

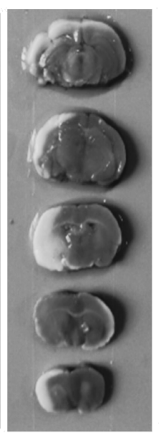

YQTL-H

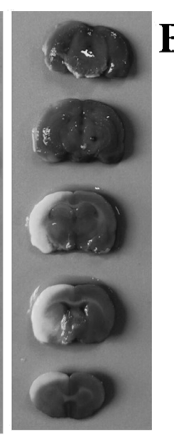

NMDP

B

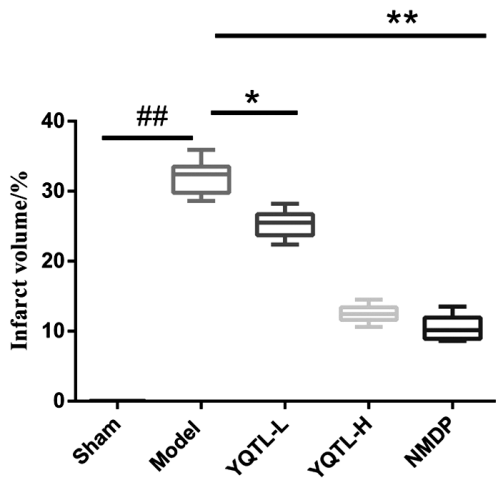

Fig. 5. Effects of YQTL on Cerebral Infarct Volume

(A) TTC staining of the cerebral infarct in rat brain. The pale area represents infarct area and red color stained area represents normal area. No brain infarction was observed in Sham group; In Model group, severe lesion was observed in both striatum and lateral cortex. Infarct volume was significantly reduced in YQTL groups and NMDP group. (B) Quantitative analysis of brain infarct volume. Sham: sham group; Model: Model group; YQTL-L: YQTL (1.8g/kg) group; YQTL-H: YQTL (3.6g/kg) group; NMDP: NMDP $(0.02 \mathrm{~g} / \mathrm{kg})$ group. ${ }^{\# \#} p<0.01 v s$. Sham group; ${ }^{*} p<0.05,{ }^{*} p<0.01 v s$. Model group. Data are presented as means \pm S.E.M. $n=8$.
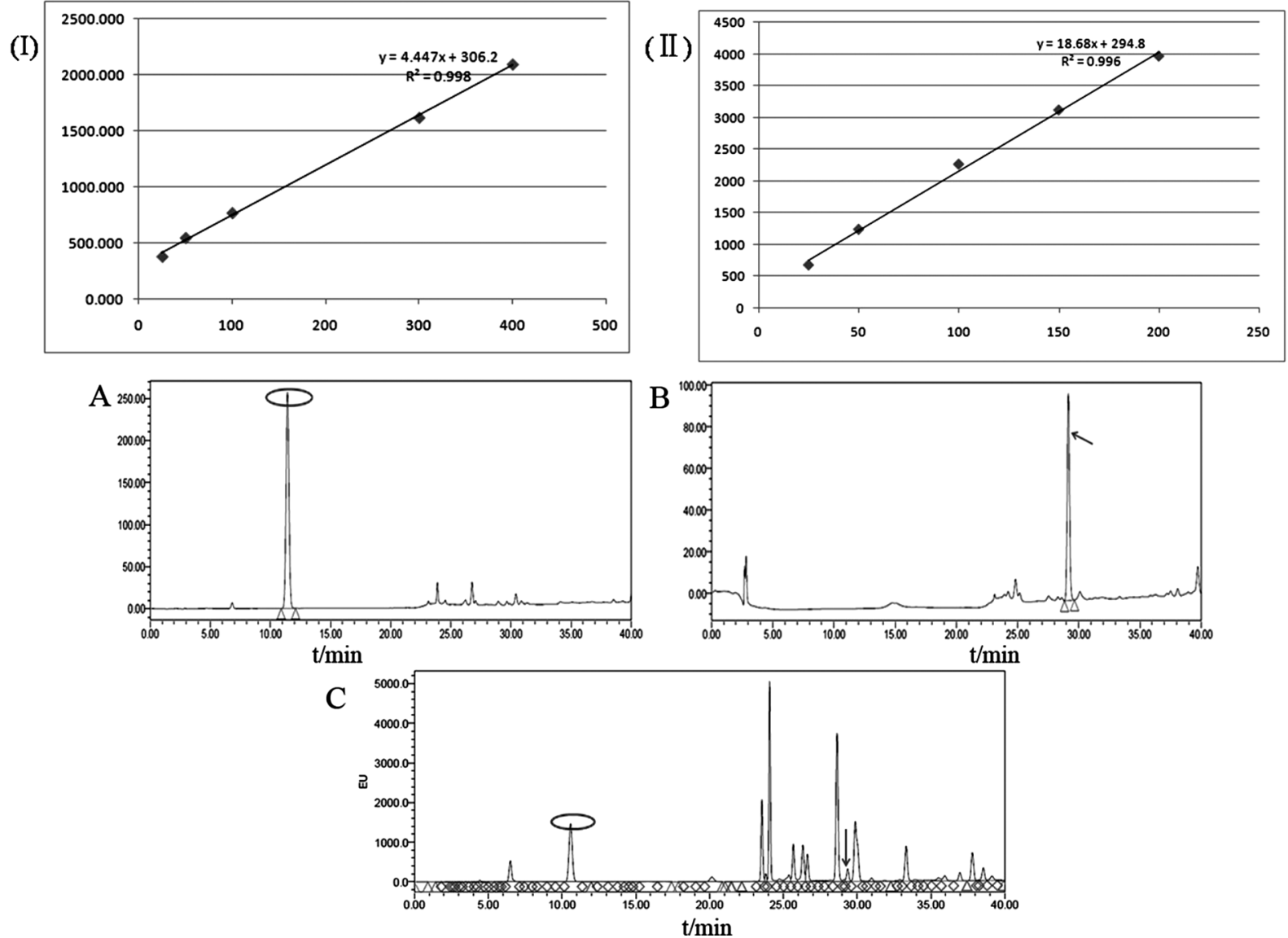

Fig. 6. Liquid Chromatograms of Glu and GABA

(I) Standard curve of Glu. $Y=4.447 X+306.2, R^{2}=0.998$ (II) Standard curve of GABA. $Y=18.68 X+294.8, R^{2}=0.9966$. (A) Glu standard preparation chromatogram detected by HPLC. Glu chromatographic peaks appeared at 11.4 min. (B) GABA standard preparation chromatogram detected by HPLC. Peaks appeared at the retention time of $29.1 \mathrm{~min}$ in the chromatomap of the standard reference material of GABA. (C) In the retention time of 11 min and $29-30 \mathrm{~min}$, liquid chromatogram of the microdialysis fluid contained YQTL appeared a peak with the same retention time as Glu or GABA standard. Ellipse and Arrow indicate chromatographic peak of Glu and GABA, respectively.

the GABA content increased to $(48.7 \pm 1.3) \mu \mathrm{mol} / \mathrm{L}$ in YQTL group $(3.6 \mathrm{~g} / \mathrm{kg})$ at reperfusion $24 \mathrm{~h}(p<0.01 v s$. Model group) (Table 2).

Drug Effect on Expression of GluN2B, ERK, p-ERK and CaMKII In order to further elucidate the potential mechanisms by which YQTL exerts its pro-survival effects on cerebral I/R injury, we evaluated the expression of proteins in the MEK/ERK signaling pathway and GluN2B, CaMKII by Western blotting, the levels of p-ERK, ERK, GluN2B and p-CaMKII proteins in the peri-infarct region of the hippocampus were examined in all groups. In Figs. 7A and B, levels of GluN2B and p-CaMKII protein in hippocampus were signifi- 
Table 1. Effect of YQTL Granules on the Content of Glu in the Striatum of I/R Rats $(x \pm \mathrm{s}, n=6)$

\begin{tabular}{cccc}
\hline \hline \multirow{2}{*}{ Group } & \multicolumn{3}{c}{ Glu $(\mu \mathrm{mol} / \mathrm{L})$} \\
\cline { 2 - 4 } & Dose $(\mathrm{g} / \mathrm{kg})$ & Ischemia $2 \mathrm{~h}$ & Reperfusion 24h \\
\hline Sham & - & $15.2 \pm 0.15$ & $17.3 \pm 1.0$ \\
Model & - & $575.1 \pm 1.05^{\# \#}$ & $268.3 \pm 1.1^{\# \#}$ \\
NMDP & 0.02 & $51.3 \pm 1.14^{* *}$ & $31.5 \pm 0.70^{* *}$ \\
YQTL-H & 3.6 & $77.3 \pm 0.76^{* *}$ & $50.3 \pm 1.56^{* *}$ \\
YQTL-L & 1.8 & $237.6 \pm 0.52^{* *}$ & $215.3 \pm 1.76^{* *}$ \\
\hline$\# p<0.01 v s$. Sham group; $* * p<0.01$ vs. Model group.
\end{tabular}

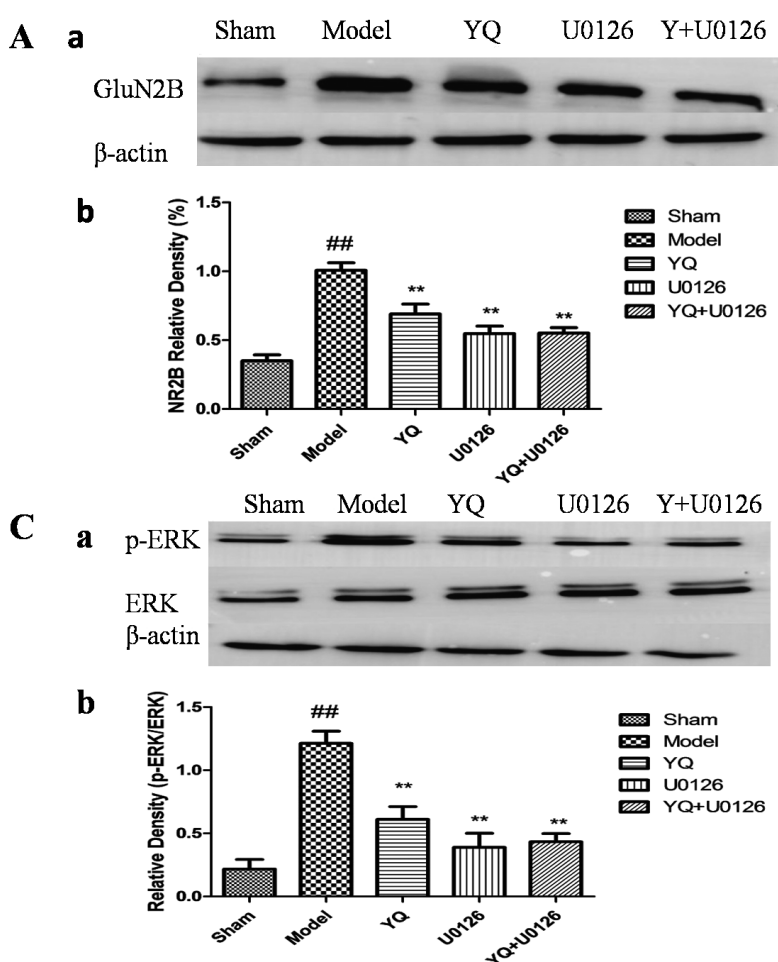

Table 2. Effect of YQTL Granules on the Content of GABA in the Striatum $(x \pm \mathrm{s}, n=6)$

\begin{tabular}{lccc}
\hline \hline \multirow{2}{*}{ Group } & \multicolumn{3}{c}{ GABA $(\mu \mathrm{mol} / \mathrm{L})$} \\
\cline { 2 - 4 } & Dose $(\mathrm{g} / \mathrm{kg})$ & Ischemia $2 \mathrm{~h}$ & Reperfusion 24h \\
\hline Sham & - & $1.15 \pm 0.25$ & $4.72 \pm 0.28$ \\
Model & - & $17 \pm 1^{\# \#}$ & $25.8 \pm 1.17^{\# \#}$ \\
NMDP & 0.02 & $36.9 \pm 1.07 * *$ & $41.9 \pm 1.1 * *$ \\
YQTL-H & 3.6 & $36.4 \pm 2 * *$ & $48.7 \pm 1.3 * *$ \\
YQTL-L & 1.8 & $23.2 \pm 3.2$ & $33.5 \pm 2.5 *$ \\
\hline$\#$ \#\# $p<0.01 v s$. Sham group; $* p<0.05, * * p<0.01 v s$. Model group.
\end{tabular}

B

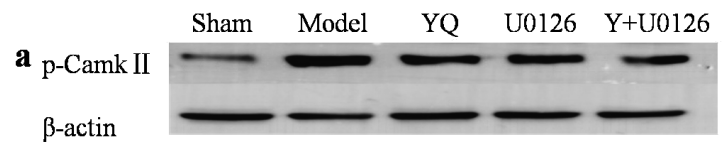

b

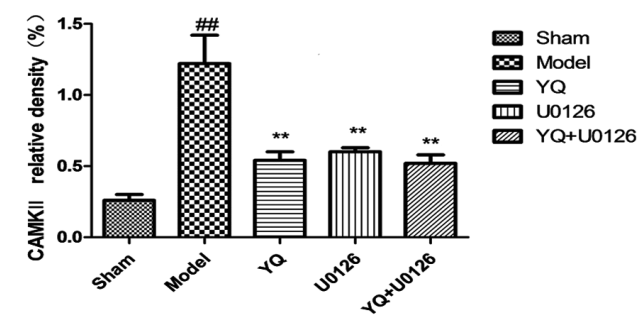

Fig. 7. Effects of YQTL on Expression Level of GluN2B, p-ERK/ERK and CaMKII

Sham: Sham group; Model: Model group; YQ: YQTL (3.6 g/kg) group; U0126: U0126 (0.05 mg/kg) group; Y+ U0126: YQTL (3.6g/kg) + U0126 (0.05 mg/kg) combine group. $\beta$-Actin was used as an internal control. (a) Representative western blots of of GluN2B, p-CaMKII, ERK and p-ERK, respectively. (b) Western blot and quantitative analysis of the expression of GluN2B, p-CaMKII, ERK and p-ERK, respectively. $(n=3)$. All the experiments were performed in triplicate. Data were expressed as mean \pm S.D. ${ }^{\# \#} p<0.01$ vs. Sham group; ${ }^{* *} p<0.01 v s$. Model group.

cantly increased by $\mathrm{I} / \mathrm{R}$, compare with Sham group $(p<0.01$ $v s$. Sham group). But YQTL remarkably suppressed the levels of GluN2B and $\mathrm{p}$-CaMKII proteins ( $p<0.01$ vs. Model group) (Figs. 7A, B). Also in Fig. 7C, YQTL remarkably reduced the levels of $\mathrm{p}$-ERK compare with Model group $(p<0.05$ $v s$. Model group). These data suggested that inhibition of $\mathrm{I} / \mathrm{R}$ injury by YQTL was associated with inhibition of the expression of NMDA receptor and MEK/ERK pathway.

\section{Discussion}

Ischemia stroke involves occlusion of blood vessels, usually occurring in the middle and anterior cerebral arteries. ${ }^{14)}$ Currently, whether the filament has been inserted into the MCA were merely judged by the distance or the sensation that the filament inserting ICA, after which the neurological score was evaluated for measuring the success of MCAO. However, the variances among the individuals were remarkable, ${ }^{15)}$ this evaluation method is not comprehensive enough. Studies have demonstrated that LDF can be used to identify the efficiency of the MCAO model. ${ }^{13)}$ Precisely, blood flow reduction is the most direct manifestation of cerebral ischemia, As shown in Fig. 2A, the cerebral blood flow rapidly dropped to $30 \%$ of the baseline when the filament reached the blood vessel, and then the blood flow was basically maintained at $30 \%$ of baseline at $2 \mathrm{~h}$ post-ischemia, blood flow was recovered to $80 \%$ after filament pulled out. Finally, recovery of cerebral blood flow was an intuitive manifestation of damage improvement, and our results showed that YQTL or NMDP could significantly improve the decline of cerebral blood flow induced by $\mathrm{I} / \mathrm{R}$ on 14th day (Fig. 2B). Although severe injuries usually occur within striatum and cerebral cortex in the MCAO model, the hippocampus is also a sensitive area of brain injury. The synaptic plasticity of hippocampus is regulated by ERK protein. ${ }^{16)}$ Therefore, we focused on the post-treatment effect of YQTL on hippocampus neurons in I/R-injured rats. Since cerebral injury peaks at $24 \mathrm{~h}$ after reperfusion, ${ }^{17)}$ we chose $24 \mathrm{~h}$ for the 
following study. The results showed that $\mathrm{I} / \mathrm{R}$ induced a larger neuronal death in the hippocampus, which was somewhat rescued by YQTL treatment implying a protective effect of YQTL on neurons under I/R conditions (Fig. 3). The content of $\mathrm{Ca}^{2+}$ increased in model group, while the concentration of $\mathrm{Ca}^{2+}$ significantly reduced in YQTL high dose group. In addition, NMDP was selected as a positive control (for calcium channel blocker), which showed better parameters on $\mathrm{Ca}^{2+}$ (Fig. 4).

The toxicity of EAA plays a key role in $I / R,{ }^{18)}$ while the massive Glu release during cerebral ischemia is the source of the excitotoxicity. As the key representation of inhibitory amino acid, GABA could obviously alleviate the excitotoxity of Glu. ${ }^{19)}$ Studies have shown that the changes of excitability and inhibitory amino acids in striatum were associated with I/R injury. ${ }^{20)}$ Therefore, antagonising EAA toxicity by inhibiting its excessive release and promoting GABA reuptake have become promising therapeutic avenues for the treatment of cerebral I/R injury. ${ }^{21,22}$ Previous studies have examined the content of amino acids in brain homogenate and cerebrospinal fluid, and the latter reflects cells that survive in the extracellular fluid, which does not really reflect the changes of amino acids. $^{23,24)}$ Microdialysis, through its continuous sampling and analysis of neurochemicals in extracellular fluids, is widely used in ischemic stroke studies. ${ }^{25}$ ) Here, our results showed that Glu levels were relatively stable in the sham group. Compared with the sham group, the Glu was rapidly increased in the model group at $2 \mathrm{~h}$ post-ischemia. The YQTL group content of Glu in the striatum was significantly lower than the model group (Table 1). Also the level of GABA significantly increased with prolonged ischemia time. And for pretreatment YQTL groups, the high dose had an obvious effect on GABA at $24 \mathrm{~h}$ post-reperfusion than NMDP (Table 2). The data confirm that the level of GABA increased with ischemia reperfusion, YQTL can accelerate the release of GABA and reduce the release of Glu to eliminate the excitatory toxicity of Glu. Taken together, the above results indicate that YQTL can protect hippocampus neurons injury induced by $I / R$ and restore the $\mathrm{rCBF}$ in rats, and this effect may be related to reduction of excitotoxicity and calcium content of two aspects through pre-treatment and post-treatment, separately. The potential mechanism of YQTL on I/R injured neurons is worth exploring.

It's worth mentioning that EAA plays an important role in maintaining the homeostasis of the central nervous system, over-expression of NMDAR is the key step leading to excitotoxicity, this excitotoxic effect is also the key to the death of neurons in the pathological state of ischemic brain injury. ${ }^{26,27)}$ As previously reported, the excessive accumulation of extracellular glutamate will over-stimulates their receptor ion channels on postsynaptic and extrasynaptic neurons. ${ }^{28)}$ However, the cellular consequences of synaptic versus extrasynaptic NMDA receptor stimulation are dramatically different. ${ }^{29)}$ This observation suggests that over-opening of NMDA receptor is no longer the only cause of excitotoxicity, and its spatial distribution may play a more important role. There is increasing evidence that the activation of extrasynaptic NMDA receptors is more important for initiating neuronal death during ischemia. ${ }^{30,31)}$ NMDAR subunit compositions have different effects on synaptic function. GluN2B is a key regulatory subunit of NMDAR, and its expression level is positively cor- related with the peak of excitatory postsynaptic potential induced by NMDAR. ${ }^{32)}$ In the adult hippocampus, GluN2Bcontaining NMDARs are located extrasynaptically, whereas activation of extrasynaptic GluN2B-containing NMDARs is coupled with cell death pathways. ${ }^{33)}$ There is evidence demonstrated that increased activation of GluN2B-containing extrasynaptic NMDARs contributes to the toxicity in neurodegenerative disorders, and $N$-acetyl-aspartyl-glutamate could protect the neurodegenerative disorders by decreasing the activity of GluN2B-containing extrasynaptic NMDARs. ${ }^{34,35)}$ In addition, studies have shown that drugs could against I/R induced injury by regulation of GluN2B expression. ${ }^{36)}$ Exactly, the $\mathrm{Ca}^{2+}$ content will increase during cerebral $\mathrm{I} / \mathrm{R}$, and $\mathrm{Ca}^{2+}$ overload can activate the auto-phosphorylation of CaMKII. As a downstream protein for GluN2B,${ }^{37)} \mathrm{p}$-CaMKII is one of the most abundant kinases in the brain and is mainly found in the hippocampus, which is not only an indicator of ischemic brain damage, but also a key enzyme in the excitatory calcium signaling pathway. ${ }^{38)}$ Therefore, down-regulating the content of p-CaMKII and GluN2B is important for the protection of cerebral I/R injury. In line with that, our result demonstrated that YQTL effectively inhibited the activation of GluN2B and p-CaMKII induced by $\mathrm{I} / \mathrm{R}$, highlighting its neuronal protective effects via direct modulation of the GluN2B and p-CaMKII expression (Fig. 7), which is consistent with the relevant studies. ${ }^{39)}$ Hence, we speculate that YQTL may protect I/R injury by effectively ameliorate extrasynaptic GluN2B-containing NMDARs mediated cell death process.

Studies have shown that excitotoxicity is closely related to the MEK/ERK pathway. ${ }^{40)}$ The MEK/ERK signaling pathway is known to be a key signaling pathway that plays critical roles in regulating neuron death and survival. Recent studies have confirmed that inhibiting the MEK/ERK signaling pathway could meliorate the cerebral I/R injury. ${ }^{41)}$ Therefore, it was important to explore whether the protective effect of YQTL is related to the ERK signaling pathway. U0126, a specific MEK inhibitor, has been shown to protect against cerebral ischemia injury, ${ }^{42}$ which was selected for comparison with YQTL to determine underlying effects of YQTL on the MEK/ERK pathway. The data from the current study showed that the level of p-ERK and ERK obviously up-regulated in $\mathrm{I} / \mathrm{R}$ exposed rats, suggesting that $\mathrm{I} / \mathrm{R}$ improved the activation of the MEK/ERK signaling pathway. However, it is worth noting that the levels of p-ERK and ERK were down-regulated in U0126 group and YQTL group. Moreover, YQTL mediated down-regulation was also promoted by U0126 (Fig. 7C). In summary, this data indicates that the inhibition of the MEK/ERK pathway may be the underlying molecular mechanism of YQTL against cerebral I/R injury, which has not been reported previously.

TCM herbal formulas play their therapeutic role through multi-targets and multi-channels. Furthermore, herbal combinations may not only act synergistically with other constituents from the same herb, but also neutralize the toxic effects of some compounds. ${ }^{43}$ ) They have been widely used to treat cardiovascular and cerebrovascular diseases in China. Although we demonstrated that YQTL could obviously against cerebral I/R injury by reducing excitotoxicity through the MEK/ERK pathway, the study has certain limitations. Firstly, to some extent, the evidence for the mechanisms involved in protection by YQTL is only relevant. Further effects of YQTL 
on neuron cells in vitro studies are merited to expand our findings. Recent studies have found that synaptic NMDARs can activate neuroprotective signal transduction pathways to improve cells death, while the activation of extrasynaptic NMDARs will lead to neuronal death. ${ }^{44}$ Finally, explore the effect of YQTL on synaptic NMDARs or select suitable inhibitor of extrasynaptic NMDARs can deepen our research.

\section{Conclusion}

YQTL can promote cerebral ischemia/reperfusion injury and restore the $\mathrm{rCBF}$, which may be associated with inhibition of excitotoxicity, overload of $\mathrm{Ca}^{2+}$ and deactivation of the MEK/ERK pathway. These findings may elucidate the pharmacological mechanism of YQTL and promote its clinical application.

Acknowledgments The work was supported by the Key Projects of Overseas Visits of Outstanding Young Backbone Talents from Universities in Anhui Province (No. gxfxZD2016117), the Academic Assistance Program for the Top-notch Innovative Talents from Universities in 2017 Provided by Anhui Province Office of Education (No. gxbjZD15), National major R\&D program matching project (Z161100002616024).

Conflict of Interest The authors declare no conflict of interest.

\section{References}

1) Li T. T., Fan M. L., Hou S. X., Li X. Y., Barry D. M., Jin H., Luo S. Y., Kong F., Lau L. F., Dai X. R., Zhang G. H., Zhou L. L., Br. J. Pharmacol., 172, 3904-3916 (2015).

2) Atef R. M., Agha A. M., Abdel-Rhaman A. A., Nassar N. N., Mol. Neurobiol., 55, 1284-1298 (2018).

3) Yang G. Y., Betz A. L., Stroke, 25, 1658-1664, discussion, 1664-1665 (1994).

4) Newcombe J., Uddin A., Dove R., Patel B., Turski L., Nishizawa Y., Smith T., Brain Pathol., 18, 52-61 (2008).

5) Olney J. W., Science, 164, 719-721 (1969).

6) Lan C. G., Zukin R. S., Nat. Rev. Neurosci., 8, 413-426 (2007).

7) Kalia L. V., Kalia S. K., Salter M. W., Lancet Neurol., 7, 742-755 (2008).

8) Léveillé F., El Gaamouch F., Gouix E., Lecocq M., Lobner D., Nicole O., Buisson A., FASEB J., 22, 4258-4271 (2008).

9) Chang J., Yao X., Zou H., Wang L., Lu Y., Zhang Q., Zhao H., $J$. Ethnopharmacol., 194, 1032-1042 (2016).

10) Zhang Y. Y., Wan H. T., Lai L. L., Yang J. H., Chen W. Y., Zhou H. F., Zhu Z. H., Yao Hsueh Hsueh Pao, 47, 1153 (2012).

11) Longa E. Z., Weinstein P. R., Carlson S., Cummins R., Stroke, 20, 84-91 (1989).

12) Ahnstedt H., Mostajeran M., Blixt F. W., Warfvinge K., Ansar S., Krause D. N., Edvinsson L., J. Cereb. Blood Flow Metab., 35, 454-460 (2015).

13) Schmid-Elsaesser R., Zausinger S., Hungerhuber E., Baethmann A., Reulen H. J., Stroke, 29, 2162-2170 (1998).

14) Candelario-Jalil E., Curr. Opin. Investig. Drugs, 10, 644-654
(2009).

15) Yanamoto H., Nagata I., Niitsu Y., Xue J.-H., Zhang Z., Kikuchi H., Exp. Neurol., 182, 261-274 (2003).

16) Pan B., Zhong P., Sun D., Liu Q., J. Neurosci., 31, 11244-11255 (2011).

17) Jia D., Han B., Yang S., Zhao J., J. Mol. Neurosci., 53, 271-279 (2014).

18) Nishizawa Y., Life Sci., 69, 369-381 (2001).

19) Han J., Wan H. T., Yang J. H., Zhang Y. Y., Ge L. J., Bie X. D., J. Asian Nat. Prod. Res., 16, 1060-1067 (2014).

20) Arranz A. M., Gottlieb M., Pérez-Cerdá F., Matute C., Neurobiol. Dis., 37, 156-165 (2010).

21) Bie X., Chen Y., Han J., Dai H., Wan H., Zhao T., Asia Pac. J. Clin. Nutr., 16 (Suppl. 1), 305-308 (2007).

22) Nuñez J. L., McCarthy M. M., Exp. Neurol., 210, 699-708 (2008).

23) Yamamoto T., Rossi S., Stiefel M., Doppenberg E., Zauner A., Bullock R., Marmarou A., "Csf and ecf glutamate concentrations in head injured patients." Springer, Vienna, 1999.

24) Sechi G. P., Petruzzi V., Rosati G., Rubattu L., Tanda F., Deiana G. A., De Riu P., Brain Res., 564, 154-158 (1991).

25) Müller M., $B M J, 324,588-591$ (2002).

26) Watanabe A., Sasaki T., Yukami T., Kanki H., Sakaguchi M., Takemori H., Kitagawa K., Mochizuki H., Neuroscience, 339, 139-149 (2016).

27) Xiao J., Kong R., Hu J., Neuroreport, 29, 723-730 (2018).

28) Karpova A., Mikhaylova M., Bera S., Bär J., Reddy P. P., Behnisch T., Rankovic V., Spilker C., Bethge P., Sahin J., Kaushik R., Zuschratter W., Kähne T., Naumann M., Gundelfinger E. D., Kreutz M. R., Cell, 152, 1119-1133 (2013).

29) Rao A., Craig A. M., Neuron, 19, 801-812 (1997).

30) Frasca A., Aalbers M., Frigerio F., Fiordaliso F., Salio M., Gobbi M., Cagnotto A., Gardoni F., Battaglia G. S., Hoogland G., Di Luca M., Vezzani A., Neurobiol. Dis., 43, 507-515 (2011).

31) Hardingham G. E., Bading H., Nat. Rev. Neurosci., 11, 682-696 (2010).

32) Loftis J. M., Janowsky A., Pharmacol. Ther., 97, 55-85 (2003).

33) Clemente A. S., Nicoll R. A., Roche K. W., Neuroscientist, 19. 62-75 (2013).

34) Jaarsma D., Veenma-van der Duin L., Korf J., J. Neurol. Sci., 127, 230-233 (1994).

35) Parsons M. P., Raymond L. A., Neuron, 82, 279-293 (2014).

36) Huang M., Cheng C., Tan H., Qin R., Zou Y., Wang Y., Zhang Y., Exp. Neurol., 295, 66-76 (2017).

37) Li Y., Sun W., Han S., Li J., Ding S., Wang W., Yin Y., Mol. Neurobiol., 54, 1-13 (2016).

38) Takano H., Fukushi H., Morishima Y., Shirasaki Y., Brain Res., 962, 41-47 (2003).

39) Vieira M. M., Schmidt J., Ferreira J. S., She K., Oku S., Mele M., Santos A. E., Duarte C. B., Craig A. M., Carvalho A. L., Neurobiol. Dis., 89, 223-234 (2016).

40) Shioda N., Han F., Fukunaga K., Int. Rev. Neurobiol., 85, 375-387 (2009).

41) Zhang J. Z., Jing L., Guo F. Y., Ma Y., Wang Y. L., Exp. Toxicol. Pathol., 59, 227-235 (2007).

42) Alessandrini A., Namura S., Moskowitz M. A., Bonventre J. V., Proc. Natl. Acad. Sci. U.S.A., 96, 12866-12869 (1999).

43) Yun Y. P., Do J. H., Ko S. R., Ryu S. Y., Kim J. H., Song H. C., Park Y. D., Ahn K. S., Kim S. H., J. Ethnopharmacol., 77, 259-264 (2001).

44) Hardingham G. E., Fukunaga Y., Bading H., Nat. Neurosci., 5, 405-414 (2002). 